\title{
The perspectives of South African academics within the disciplines of health sciences regarding telehealth and its potential inclusion in
}

\section{student training}

\author{
S M Govender, MComm Path (UKZN), M Mars, MB ChB (UCT) \\ Department of TeleHealth, Nelson R Mandela School of Medicine, University of KwaZulu-Natal, Durban, South Africa
}

Corresponding author: S M Govender (Samantha.govender@smu.ac.za)

\begin{abstract}
The professional training and development of healthcare professionals in the area of telehealth is important to ensure the sustainability of this service delivery model. Tertiary institutions are among the key constituents involved in telehealth education, training and development. Academics within the healthcare sciences should therefore have the necessary experience and knowledge in this area to support the education and training of students. The objectives of this study were to determine the perspectives, experiences and attitudes of South African academics within various disciplines of health sciences regarding telehealth, as well as their views on suitable content areas for a telehealth module. A descriptive survey design was implemented. Sixtysix fulltime employed academic staff from five universities participated. The majority of participants were familiar with the terms telehealth/electronic health (eHealth), while 59\% were unfamiliar with terms such as synchronous and asynchronous services. Eighty percent of respondents felt it necessary to include telehealth in the curriculum. The majority (89\%) did not conduct research in telehealth. Seventy-one percent felt positive that telehealth could benefit the profession, and $30 \%$ stated that lack of standards creates a negative attitude toward the area and its sustainability. The majority of participants (77\%) felt that their final-year students knew very little about telehealth upon exiting their study programme. Almost half (45\%) of the participants felt that ethical issues were the most important aspect that needed to be included in a telehealth module, while data management was ranked as being least important (49\%). The correlation between the perspectives on ethical issues and limitations to telehealth was statistically significant $(p=0.007)$, implying that participants saw lack of ethical considerations as a limitation to the uptake of telehealth practice. While attitudes regarding telehealth were positive, concerns were raised around the lack of standards and guidelines. Opportunities for professional development in telehealth need to be created through continued professional development (CPD) workshops and training. This in turn may provide more skilled faculty to teach in this area, allowing students to receive better instruction on telehealth service delivery models.
\end{abstract}

Afr J Health Professions Educ 2018;10(1):38-43. DOI:10.7196/AJHPE.2018.v10i1.957

The words telemedicine and telehealth are used synonymously by some. Telemedicine refers to the use of information and communication technologies for clinical diagnosis and monitoring and the provision of healthcare over distance, but telehealth is a broader concept. Telehealth is defined by the Health Resources and Services Administration ${ }^{[1]}$ as 'the use of electronic information and telecommunications technologies to support long-distance clinical healthcare, patient and professional health-related education, public health and health administration' Telehealth has been viewed as a promising method of addressing the current health challenges surrounding service delivery to remote and rural areas. It can be used to alleviate the shortage of healthcare practitioners; improve access to specialist physicians; reduce the costs of accessing healthcare services by reducing the need to travel for consultation; and provide support to rural general practitioners and community service therapists, thereby improving retention in rural communities. ${ }^{[2-6]}$

One of the suggested strategies to promote telehealth at a national level within developing countries is to introduce telehealth into the education and training programmes of healthcare professionals, both undergraduate and postgraduate, so that they are aware of, and can use, telehealth methods to provide healthcare to their patients. ${ }^{[7]}$ The benefits associated with telehealth provide impetus for student and professional training across the healthcare disciplines. As students receive their training from academics at tertiary institutions, trained faculty with relevant content knowledge ${ }^{[8,9]}$ and research experience in the field ${ }^{[10]}$ should be available to disseminate this information and to demonstrate how telehealth can be used to provide and improve patient care.

A lack of skilled personnel to facilitate training of healthcare professionals is a barrier to sustaining telehealth models of service delivery. ${ }^{[1]}$ A study on telehealth in primary care found that telehealth could support the primary healthcare approach, as its inclusion into student training can be used to connect students with patients from remote and rural areas, allowing them access to diverse communities, but also that insufficient training and exposure during undergraduate training contributed to a limited uptake of this technology. ${ }^{[12]}$ These results are especially relevant in South Africa (SA), where almost $50 \%$ of the population lives in rural areas. ${ }^{[13]}$

There is sufficient evidence supporting the use of telehealth services to improve patient care across many disciplines of health, including medicine, physiotherapy, nursing, audiology and speech-language pathology. ${ }^{[6,14-19]}$ However, there is a paucity of literature pertaining to the training and education of students in using and implementing telehealth services. A systematic review conducted by Edirippulige and Armfield ${ }^{[20]}$ found no record of education and training programmes on telehealth in Africa. This 
is of concern, as the importance of telemedicine has long been recognised by the SA government. The first phase of the SA National Telemedicine System was implemented in 1999, but was not successfully sustained. ${ }^{[21]}$

Healthcare acts and policies within SA recognise the value of information and communication technology (ICT) in health, and support the use of telehealth applications and technology within the healthcare service delivery model. ${ }^{[22-24]}$ The National Department of Health outlines 10 priorities (the 10-point plan) within the national service delivery agreement, one of which is the need to improve health infrastructure, including the use of ICT and sophisticated technology to advance patient care, and has developed a National eHealth Strategy. ${ }^{[22]}$ One aspect identified within the strategic objectives is the need to improve telehealth capacity building. The strategy notes that educational opportunities in telehealth are limited, and government therefore aims to promote capacity development in telehealth through education and research. Universities, through their academic staff, have been identified as key role-players to facilitate this process. Related to this is the development of education and training courses that are well structured, to provide the theoretical and practical competencies required for administering clinical and educational services via a telehealth model. In order to capacitate academics to teach and train students in this area, key aspects relating to telehealth need to be understood.

A review of the literature identified key knowledge areas required for effective telehealth practice that should be included in a telehealth course: computer competence and literacy; $;^{[3,25,26]}$ understanding of ethical and legal issues; ${ }^{[25-29]}$ understanding of the protocols and standards that guide good practice; ${ }^{[30]}$ and data management, specifically relating to online transmission, retrieval and storage of data. ${ }^{[29]}$ It is important to put these issues into the SA context in view of current healthcare constraints, service delivery issues and the infrastructure requirements of a telehealth service.

Early literature from the developed world found that some disciplines of healthcare reported limited or no exposure to telehealth during their undergraduate training. In 2002, the American Speech and Health Association found that only $11 \%$ of the 1667 American speech language pathologists and audiologists used telehealth in their practice. Lack of theoretical and clinical exposure to telehealth at undergraduate level, lack of guidelines and insufficient clinical evidence were cited as the primary reasons for non-use by over three-quarters of respondents. ${ }^{[31]}$ The current situation in SA is believed to be similar.

The perspectives, practices and overall attitudes of academics toward education and training in telehealth is considered a key enabler of sustainable development of telehealth. ${ }^{[32]}$ The aim of this study was to determine the perspectives, attitudes and exposure of academics within the various disciplines of the health sciences to telehealth and its inclusion in student training. The study further aimed to identify telehealth information that exists within the current curricula as well as to understand which areas of telehealth academics consider important when designing a telehealth course.

\section{Methods}

Ethical approval to conduct the study was obtained from the University of KwaZulu-Natal Ethics Committee (ref. no. HSS/0335/014D). A descriptive survey design was implemented, with quantitative methods of analysis. The questionnaire developed by the American Speech and Hearing Association ${ }^{[31]}$ was adapted to include questions relevant to the SA context.
The questionnaire comprised 30 questions across four domains linked to the objectives of the study, which were to determine SA academics':

(i) experiences (in teaching, learning and research) with telehealth;

(ii) attitudes about telehealth; and

(iii) perspectives on what they considered would be most valuable for inclusion in a telehealth course, based on six key areas provided.

The questionnaire was circulated electronically via Google forms. Questions and statements were multiple choice, yes-or-no responses or open-ended, allowing participants to explain their responses.

Letters requesting participation were sent to the seven SA universities offering health sciences programmes, of which five consented to participate in the study. Once institutional permission from the university was granted, permission from heads of department (HODs) of the various disciplines was requested. Some HODs failed to respond to the request, and this contributed to a low response rate. Invitations to participate in the study could only be sent to the list of email addresses that could be obtained from the HODs who furnished this information. An information letter together with a consent form and a link to the questionnaire was emailed to 170 academic staff members within health sciences departments from the five participating universities. The online survey system allowed the participants 3 weeks to respond.

In addition to basing our questionnaire on one that had already been developed and used, other measures to ensure the validity of our questionnaire included a pilot study. Ten part-time employed academics were asked to complete the questionnaire to discover whether they experienced any problems answering any of the questions. They were required to complete a response form giving feedback on the clarity of questions, language and grammar, as well as on the length of the questionnaire. No-one experienced any challenges, and no changes were made to the questionnaire. The data were analysed using descriptive and inferential statistics. For the 6-point Likert-scale questions on key areas that could be included into a telehealth module, Spearman's correlation coefficient was calculated. The mean square contingency coefficient, the phi coefficient, was used to determine the degree of association between the binary variables (yes-or-no responses). Alpha was set at 5\%.

\section{Results}

A total of 66 academics completed the questionnaire, a response rate of $39 \%$. The distribution of participants across the various health science disciplines is shown in Fig. 1.

Twenty-nine of the academics ( $44 \%$ ) had $>10$ years of teaching experience, 19 had $<5$ years of experience (29\%) and the remaining 18 had between 5 and 10 years of experience (27\%).

\section{Experience with and exposure to telehealth (teaching, learning and research)}

Participants were asked about their experiences with, and exposure to, telehealth. The questions related to their understanding of key definitions and operation and familiarity with telehealth equipment, and their involvement in teaching and research within these areas. Regarding how they were first introduced to telehealth, 51 respondents (77\%) indicated that they had read about it, while $9(14 \%)$ had heard about it during a conference presentation. The remainder were introduced to it by colleagues 


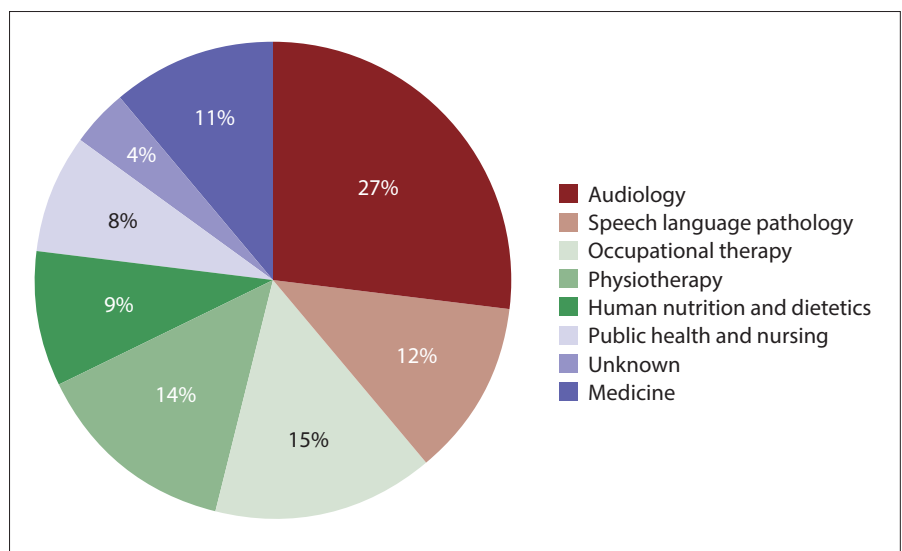

Fig. 1. Distribution of participants by discipline, \%.
One person who felt that it was unnecessary to include telehealth in the curriculum, however, added:

'Our students are being trained to be clinicians and not academics, the use of telehealth between client and clinician is not relevant in our underresourced areas ... among clinicians it is used, and can be learnt in the field at the particular venue, and hence does not to be included in the curriculum' (occupational therapist, 7 years' experience).

With regard to devices and technology, all institutions had some form of telehealth equipment across the various disciplines. The three disciplines reporting the most access to technology and devices were audiology, physiotherapy and nursing. Three of the five institutions owned a Kudu-wave 5000 tele-audiology device. One institution is involved in mHealth (mobile health) and has developed a smartphone application for hearing screening.

Table 1. Experiences with and exposure to telehealth (teaching, learning and research) $(N=66)$

\begin{tabular}{lll}
\hline & Yes, $\boldsymbol{n}(\%)$ & No, $\boldsymbol{n}(\%)$ \\
\hline Familiarity with basic telehealth terminology & $59(89)$ & $7(11)$ \\
Familiarity with synchronous v. asynchronous telehealth services & $27(41)$ & $39(59)$ \\
Attended a CPD-related activity on telehealth & $18(27)$ & $48(73)$ \\
Experience with operating a telehealth device & $26(39)$ & $40(61)$ \\
Researching telehealth & $7(11)$ & $59(89)$ \\
Teaching telehealth & $14(21)$ & $52(79)$ \\
Do you think telehealth should be included in the curriculum? & $53(80)$ & $10(15)$ \\
CPD continuing professional development. & & $3(5)$
\end{tabular}

and demonstrations conducted by suppliers. Responses to the yes-or-no options are shown in Table 1.

The majority of the academics did not teach (79\%) or research $(89 \%)$ telehealth. There was a statistically significant relationship between teaching and research ( $p=0.001)$, as those who were teaching telehealth were also conducting research in the area.

The majority (80\%) of participants indicated that they felt it is necessary to include telehealth in the curriculum, while some were either unsure or disagreed. An open-ended question asked them to support their response. Most attributed their reasoning to meeting the needs of communities through the use of telehealth services, and 35 (53\%) participants stated that students need to have knowledge, exposure and competence in this area so that it is sustainable. One participant stated:

'The times that we live in are changing and platforms for service delivery are also changing. Students should be able to function in various contexts and use various platforms to offer services to patients. I think understanding tele-audiology will be beneficial to students, especially taking into consideration the shortage of professionals in SA' (audiologist and speech-language pathologist, 8 years' experience).

The importance of using internet-based services was emphasised:

'Telehealth is a collection of means or methods for enhancing healthcare, public health, and health education delivery and support using telecommunications technologies. Telehealth encompasses a broad variety of technologies and tactics to deliver virtual medical, health and education services. I believe that content is freely available to all via the internet and it is best to incorporate it into teaching rather than avoid it' (physiotherapist, 5 years' experience).
The discipline of nursing across two institutions reported management of rural community patients via telehealth services.

\section{Attitudes towards and perspectives on telehealth}

A total of $47(71 \%)$ respondents indicated that they felt that telehealth could positively benefit their profession, and $47(71 \%)$ stated that it has the potential to address service delivery barriers. Twenty (30\%) stated that the lack of standards for telehealth practice creates a negative attitude towards the area, while 11 (17\%) had a negative attitude regarding the sustainability of telehealth practices even though they felt positive about the benefits. A total of $46(70 \%)$ felt that introducing content on telehealth-based teaching resources could improve overall teaching and learning. However, 18 (27\%) did not feel that introducing it would improve learning outcomes for their respective degrees. The responses to the other statements are shown in Table 2.

There was no correlation between responses to the question, 'Can telehealth positively impact the profession?' and the question on lack of standards $(p=0.369)$. This implies that not all participants who felt that the lack of standards, guidelines and policy makes it difficult to implement telehealth thought that this would interfere with the positive impact that telehealth could make on their profession.

Additional comments regarding their attitudes towards telehealth were sought, and included the following:

'More information and practical demonstrations may change negative views of most audiologists, including academics, toward tele-audiology' (audiologist, 20 years' experience).

'I don't know much about tele-audiology currently and would benefit from knowing more' (audiologist and speech-language pathologist, 8 years' experience). 
'It is unrealistic at most district-level facilities around our country, and so will students actually be able to use or benefit from this while studying or when qualified?' (occupational therapist, 1 year's experience).

'I believe in its potential to resolve many of the practical issues we experience in training interns (human nutrition and dietetics, 20 years' experience).

Respondents were asked whether they thought that telehealth-based services would be a feasible way of ensuring that students have adequate central to their profession and their selection of ethical issues as most relevant was also significant $(p=0.04)$.

\section{Discussion}

Telehealth can improve service delivery to remote and rural areas, reduce health service disparities that exist between socioeconomic groups and reduce health costs. Education and training in this area would strengthen the health system's capacity to deliver and sustain these services. ${ }^{[20]}$ Academics are central in facilitating the education and training of undergraduate healthcare professionals. The key findings of this study are that the majority of participants do not have much experience with or knowledge about telehealth, do not include any telehealth content in their teaching, have not used a telehealth device and do not have any current research interests in this area. This relationship between teaching and research in telehealth was significant, implying that those participants who were teaching in the area were also engaged in research, possibly as a way to advance their knowledge.

The academics' lack of knowledge about telehealth makes it difficult for them to teach in

Table 2. Attitudes regarding telehealth $(N=66)$

\begin{tabular}{|c|c|c|}
\hline Statement & Yes, $n(\%)$ & No, $n(\%)$ \\
\hline Telehealth can positively impact our profession & $47(71)$ & $19(29)$ \\
\hline Lack of standards, guidelines and policy makes it difficult to implement such practice & $20(30)$ & $46(70)$ \\
\hline Face-to-face contact is central to our professional interaction, making tele-audiology inappropriate & $10(15)$ & $56(85)$ \\
\hline Telehealth can address the barriers to services related to access and language between clinician and patient & $47(71)$ & $19(71)$ \\
\hline Telehealth can improve health service delivery in SA & $47(71)$ & $19(71)$ \\
\hline Telehealth is a promising concept, provided that a structured curriculum is designed to train students appropriately & $50(76)$ & $16(24)$ \\
\hline I think that telehealth is sustainable within the SA context & $32(48)$ & $34(52)$ \\
\hline $\begin{array}{l}\text { Introducing telehealth into clinical training would improve learning outcomes by increasing exposure to more diverse patients } \\
\mathrm{SA}=\text { South Africa. }\end{array}$ & $48(73)$ & $18(27)$ \\
\hline
\end{tabular}

exposure to a maximum number and variety of patients, of whom $55(83 \%)$ agreed. When asked whether their students were knowledgeable about telehealth services, 51 (77\%) felt that their students knew very little about them. Only $14(21 \%)$ indicated that the introduction of telehealth modules was discussed in curriculum planning meetings.

Finally, academics were asked to rank, in order of importance from most important to least important, the six content areas considered relevant for a telehealth module identified from the literature. These were: standards and protocols; ethical issues; computer literacy and understanding of computers and technology; limitations of telepractice; telepractice as it relates to the SA and African context; and data management as it relates to online service delivery. Only 54 participants responded to this question in full (Fig. 2).

A significant correlation was noted between the variables 'ethical issues' and 'limitations of telehealth services' $(p=0.007)$. Respondents who regarded ethical issues as most or very important also regarded limitations of telehealth services to be very important or important. The correlation between individuals' responses to the question regarding how face-to-face contact is

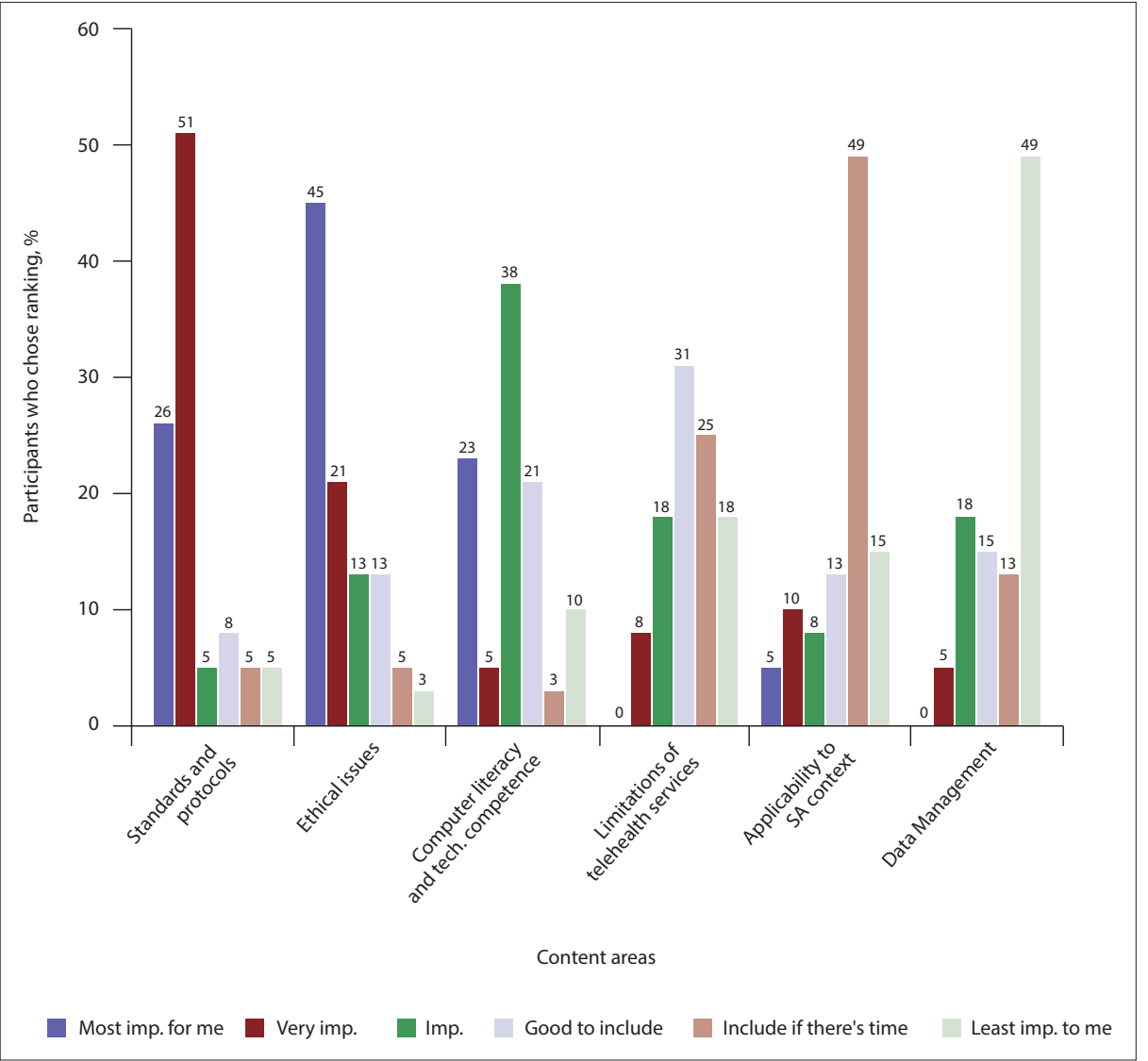

Fig. 2. Participant rankings of importance (imp.) of six content areas for a telehealth module (6-point Likert scale). 
this area. This finding supports a recent study by Grogan-Johnson et al., ${ }^{[29]}$ who surveyed telepractice training in graduate speech-language pathology and audiology programmes. The barriers to adopting telepractice in graduate programmes were related to, among others, a lack of trained faculty to provide instruction. Ehnfors and Grobe ${ }^{[33]}$ describe this as a real challenge facing healthcare professionals who may find themselves in the future working in a technologically driven healthcare system without the necessary competencies. These studies provide strong motivation for education and training, and demonstrate the need for certification programmes for both academics and healthcare professionals, so that students can also be the recipients of this information.

Although the majority of academics displayed a positive attitude towards the impact that telehealth can make on the profession, and on the improvement of service delivery, the lack of available standards and guidelines created a negative attitude towards it. $\operatorname{Picot}^{[25]}$ emphasises the need for guidelines and standards to be developed across all professions that intend using telehealth systems and methods, and further recommends that education and training standards be developed so that suitable knowledge and skills can be obtained. A systematic review by Molini-Avejonas et al. ${ }^{[34]}$ identified the barriers to the use of telehealth in the speech, language and hearing sciences as the lack of training, regulation of practice and acceptance and recognition of telehealth benefits by both the public and professionals. These findings further support the need for training and development in the area of telehealth.

In an article by Frenk et al. ${ }^{[35]}$ on transforming education for health professionals to strengthen health systems, it was emphasised that ICT is important for transformative learning in terms of exposing undergraduates to telehealth models of service delivery. The authors state that 'an exciting area of development is the application of ICT to build global consortia of education and institutions to leverage their resources, realise synergies and transform educational opportunity into a global public good'. A survey of the attitudes of 202 audiologists toward tele-audiology identified interest in using internet-based facilities to provide patient support. However, participants had concerns regarding their lack of knowledge of and exposure to technologically based services during their undergraduate years. ${ }^{[36]}$ The literature also shows that a lack of user acceptance of technology is a primary reason for the poor uptake of telehealth. ${ }^{[37,38]}$ Other studies suggest that exposure to and experience with telehealth increases positivity. ${ }^{[36,39]}$

The present study reflects an overall lack of experience with and exposure to telehealth in SA academics. In addition, participants demonstrated varied attitudes regarding the feasibility and sustainability of telehealth within their contexts. This attitudinal disposition could shape learners' interest and practice within the area. The majority of participants did not feel confident in the subject matter, owing to their lack of exposure to it. A way forward in addressing this would be to promote professional development activities in the area of telehealth. Various systematic reviews indicate that across the different health science disciplines, there are needs for protocol and guideline development, increasing confidence and competence, and the development of training standards. ${ }^{[25,29,40,41]}$ This is in keeping with the strategic priorities of the SA government, in terms of the eHealth strategy for SA. ${ }^{[22]}$ According to the World Health Organization, ${ }^{[42]}$ health professionals and academic institutions are among the key constituents involved in addressing the health needs of communities, through evidencebased teaching and the development of new and improved methods of service delivery. ${ }^{[42]}$
For the purposes of the study, six key areas were suggested as potential content areas for a telehealth module. Protocol and standards development was ranked as the most important area by participants. This was seen to be one of the key strategies necessary for promoting the advancement of telehealth in various health disciplines, as well as for interprofessional collaboration. ${ }^{[43]}$ Wade et al. ${ }^{[44]}$ have also identified the development of protocols as one of the key components of sustainable practice.

Contextual relevance is important within healthcare, especially when one considers establishing infrastructure and rolling out technology to promote health services. In a context where social determinants of health have contributed to the inequitable distribution of health resources and access, ${ }^{[45]}$ and to failure in redressing the injustices of the past, careful consideration must be taken to ensure that these service delivery models are both feasible and sustainable. Respondents did not feel that contextual relevance was an important component of a telehealth module, with $49 \%$ stating that it can be included if there is time.

Data management was viewed as the least important subject to be included in a telehealth module. However, data management is a very important part of telehealth services, especially when one considers the intricacies involved in the storage, retrieval and transmission of patient information. Failure to adequately manage patient data could result in malpractice. Data management was, however, ranked as the least important component by $49 \%$ of respondents.

An understanding of the legal and ethical issues related to telehealth service delivery is crucial for effective practice. ${ }^{[27]}$ Ethical practice guides professional behaviour, and is central to service delivery. Its importance was reflected in the responses, with $45 \%$ rating it as the most important component of a telehealth module, and $21 \%$ as very important. A significant correlation was noted between the variables 'ethical issues' and 'limitations', as well as between face-to face-contact and ethical practice. This implies that participants considered the limitations of telehealth practice and face-toface contact with patients as important ethical considerations.

According to Hebert et al., ${ }^{[46]}$ an understanding of technology, together with its advancement and development, is absolutely integral in promoting the progression of the science behind telehealth. They emphasise that it is important to also understand how the patient views technology and its ability to assist them in healthcare. This understanding is largely developed from the information imparted by a knowledgeable healthcare provider. Understanding how technology works is important, considering that telehealth technology can range from simple videoconferencing technology to sophisticated computer programs and virtual environments. ${ }^{[47]}$ Jobson $^{[48]}$ mentions that while the government in the USA has significantly progressed in providing medical technology and information systems to support the healthcare system, a lack of trained professionals has resulted in its underutilisation. Computer literacy and competence in using technology was ranked third of the six components.

The limitations of this study were the refusal of two universities to participate, and the small sample size, representing less than $50 \%$ of medical staff at the five participating universities, therefore limiting the generalisability of study findings. The requirement to rank the six content areas on an ordinal scale of importance may give a skewed impression of the overall view of the respondents. The relative differences in importance are not known. Some respondents, for example, may have considered two or more components to be of very similar importance, but were obliged to rank them. 


\section{Conclusion}

The role of academia within telehealth education and training is emphasised in the literature, and relates to the need for its inclusion in teaching and clinical training for its sustainability. Academics in this study shared the view that the inclusion of telehealth in the curriculum could be beneficial for students, and for their own development. They also considered the development of standards and protocols and legal and ethical issues as the most important areas to include in a telehealth course, while applicability to the SA and African context and data management were viewed as the least important considerations.

A dialogue needs to begin among the various disciplines on how to integrate telehealth knowledge and clinical training into their curricula. Academics are at the forefront of providing knowledge to students, but can only do so if they are knowledgeable themselves. It would also be valuable for more research to be conducted within individual health professions regarding the clinical implications of introducing telehealth into curricula. This will increase knowledge production, which could lead to knowledge translation, thus ultimately addressing the knowledge-to-action gap.

This study also highlights the need to develop training standards and guidelines for telehealth. Guidelines - clinical, technical, operational and ethical - are required to ensure quality of care and to overcome current negative perceptions of telehealth. Professional development in the area of telehealth for academics is also required, and can be facilitated through more workshops, conference presentations given by experts in the field and demonstrations by companies selling telehealth systems. The future of telehealth services depends largely on the pursuit of high-quality training and development, as it is difficult to envisage the use of technologically based healthcare without transforming the training of healthcare professionals.

1. Health Resources and Services Administration. Defining Telehealth in Policy. https://www.hrsa.gov/sites/default/ files/hrsa/advisory-committees/rural/publications/2015-telehealth.pdf (accessed 4 June 2017).

2. Bonney A, Knight-Billington P, Mullan J, et al. The telehealth skills, training and implementation project: An evaluation protocol. JMIR Res Protoc 2015;4(1): e2. https://doi.org/10.2196/resprot.3613

3. Carter L, Horrigan J, Hudyma S. Investigating the educational needs of nurses in telepractice: A descriptive exploratory study. J Univ Contin Edu 2010;36(1):1-20. https://doi.org/10.21225/D5RP4B

4. Givens GD, Elangovan S. Internet applications to tele-audiology - nothing but net. Am J Audio 2003:12(2);59-65 5. Yao J, Yao, D, Givens GD. A browser-server-based tele-audiology system that supports multiple hearing tes modalities. Telemed E Health 2015;21(9):697-704. https://doi.org/10.1089/tmj.2014.0171

6. Swanepoel DW, Olusanya BO, Mars M. Hearing healthcare delivery in sub-Saharan Africa - a role for teleaudiology. J Telemed Telecare 2010;16(2):53-56. https://doi.org/10.1258/jtt.2009.009003

7. Edirippulige S, Armfield, NR, Smith A. A qualitative study of the careers and professional practices of graduates from an e-health postgraduate programme. J Telemed Telecare 2013;18(8):455-459. https://doi.org/10.1258/jtt.2012. from an
gth 107

8. Rena, U. Who will teach: A case study of teacher education reform. San Francisco: Caddo Gap Press, 2000.

9. Rice JK. Teacher quality: Understanding the effectiveness of teacher attributes. Washington, DC: Economic Policy Rice JK. Teacher

10. Shulman LS. Those who understand: Knowledge growth in teaching. Edu Res 1986;15(2):4-14.

11. Conde JG, De S, Hall RW, Johansen E, Meglan D, Peng GC. Telehealth innovations in health education and training Telemed J E Health 2010;16(1):103-106. https://doi.org/10.1089\%2Ftmj.2009.0152

12. Flynn D, Gregory P, Makki H, Gabbay M. Expectations and experiences of eHealth in primary care: A qualitative practice-based investigation. Int J Med Inform 2009;78(9):588-604. https://doi.org/10.1016/j.jimedinf.2009.03.008 13. Statistics South Africa. Technical report. Community survey 2016. http://www.statssa.gov.za/?p=9922 (accessed June 2017).

14. Bashshur RL. Telemedicine and health care. Telemed J E Health 2002;8(1):5-12. https://doi. org/10.1089/15305620252933365

15. Boots RJ, Singh S, Terblanche M, Widdicombe N, Lipman J. Remote care by telemedicine in the ICU: Many models of care can be effective. Cur Opin Crit Care 2011;17(6)634-640. https://doi.org/10.1097/ MCC.0b013e32834a789a

16. Jin, C, Ishikawa A, Sengoku Y, Ohyanagi, T. A telehealth project for supporting an isolated physiotherapist in a rural community of Hokkaido. J Telemed Telecare 2000;6(Supp 2):S35-S37.
17. Tousignant M, Moffet H, Cabana F, Simard J. Patients' satisfaction of healthcare services and perception with in-home telerehabilitation and physiotherapists' satisfaction toward technology for post-knee arthroplasty. An embedded study in a randomized trial. Telemed J EHealth 2011;17(5):376-382. https://doi.org/10.1089 tm. 2010.0198

18. Dansky KH, Joseph V, Bowles K . Use of telehealth by older adults to manage heart failure. Res Gerontolog Nurs 2008;1(1):25-32. https://doi.org/10.3928/19404921-20080101-01

19. Fu S, Theodoros DG, Ward EC. Delivery of intensive voice therapy for vocal fold nodules via telepractice: A pilot feasibility and efficacy study. J Voice 2015;29(6):696-706. https://doi.org/10.1016/j.jvoice.2014.12.003

20. Edirippulige S, Armsfield NR. Education and training to support the use of clinical telehealth: A review of the literature. J Telemed Telecare 2016;23(2):273-282. https://doi.org/10.1177/1357633X16632968

21. Mars M. Tele-education in South Africa. Frontiers Pub Health 2014;2(173):1-10. https://doi.org/10.3389/ fpubh.2014.00173

22. Department of Health, South Africa. eHealth strategy for SA (2012 - 2016). Pretoria: NDoH, 2012. http://www health-e.org.za/wp-content/uploads/2014/08/South-Africa-eHealth-Strategy-2012-2017.2012.pdf (accessed 3 Augus 2016).

23. South Africa. National Health Act No. 61 of 2003 .

24. Government of South Africa. Negotiated Service Delivery Agreement for Outcome 2. A Long and Healthy Life for All South Africans, 2010. Pretoria: Government Printer, 2010. http://www.kznhealth.gov.za/summit/outcome2.pd (accessed 4 June 2016).

25. Picot $J$. Meeting the need for educational standards in the practice of telemedicine and telehealth. J Telemed Telecare 2000;6(2):59-62. https://doi.org/10.1258/1357633001935608

26. Lamb GS, Shea K. Nursing education in telehealth. J Telemed Telecare 2006;12(2):55-56. http://do org/10.1258/135763306776084437

27. George C, Whitehouse D, Duquenouy P. eHealth: Legal, Ethical and Governance challenges. Berlin, London: Springer, 2013.

28. Fleming DA, Edison KE, Pak H. Telehealth ethics. Telemed J e-Health 2009;15(8):797-803. https://doi.org/10.1089/ tmi. 2009.0035

29. Grogan-Johnson S, Meehan R, McCormick K, Miller N. Results of a national survey of preservice telepractice training in graduate speech-language pathology and audiology programs. J Contemp Issues Commun Sci Disord 2015;42:122-137. http://doi.org/1092-5171/15/4201-0122

30. Grol R, Grimshaw J. From best evidence to best practice: Effective implementation of change in patient care. Lancet 2003;362(9391):1225-1230. https://doi.org/10.1016/S0140-6736(03)14546-1

31. American Speech-Language-Hearing Association. Survey Report on Telepractice Use among Audiologists an Speech-Language Pathologists. https://www.asha.org/uploadedFiles/practice/telepractice/Surveyof Telepractice.pd (accessed 20 March 2016).

32. Dean CM, Stark AM, Gates CA, et al. A profile of physiotherapy clinical education. Aus Health Review 2009;33(1):38-46. Dean CM, Stark AM, Gates CA, et al. Profile of physiotherapy clinical education. Aus Healt Review 2009;33(1):38-46. https://doi.org/10.1071/AH090038

33. Ehnfors M, Grobe SJ. Nursing curriculum and continuing education: Future directions. Int J Med Inform 2004;73(78):591-598. https://doi.org/10.1016/.i.imedinf.2004.04.005

34. Molini-Avejonas DR, Rondon-Melo S, Amato CA, Samelli AG. A systematic review of the use of telehealth in speech, language and hearing sciences. J Telemed Telecare 2015;21(7):367-376. https://doi.org/10.1177/1357633X15583215

35. Frenk J, Chen L, Bhutta ZA, et al. Health professionals for a new century: Transforming education to strengthen health systems in an interdependent world. Lancet 2010;376(9756):1923-1958. https://doi.org/10.1016/S0140 6736(10)61854-5

6. Singh G, Pichora-Fuller MK, Malkowski M, Boretzki M, Launer S. A survey of the attitudes of practitioners toward teleaudiology. Int J Audiol 2014;53(12):850-860. https://doi.org/10.3109/14992027.2014.921736

37. Nwabueze SN, Mesa NP, Kifle M, Okol C, Chustz M. The effects of culture of adoption of telemedicine in medically underserviced com Ja

38. Pagliari C Sloan D, Gregor P et al What is eHealth. A scoping exercise to map the field. I Med Int Res 2005; 7(1):201210. https://doi.org/10.2196\%2Fjmir.7.1.e9

39. Hanson D, Calhoun J, Smith D. Changes in provider attitudes toward telemedicine. Telemed J E Health 2009;15(1):39-43. https://doi.org/10.1089//jmi.2008.005

40. Mahomed F, Swanepoel DW, Eikelboom RH, Soer M. Validity of automated threshold audiometry: A systematic review and meta-analysis. J Ear Hearing 2013;34(6):745-752. https://doi.org/10.1097/AUD.0b013e3182944bdf

41. Govender SM, Mars M. The use of telehealth services to facilitate audiological management for children: A scoping review and content analysis. J Telemed Telecare 2017;23(3):392-401. https://doi.org/10.1177/1357633X16645728

42. World Health Organization. Cited in Telemedicine: Opportunities Member States, 2012. Report on the Second Global Survey on eHealth 2009 (Global Observatory for eHealth Series, Volume 2). Geneva: WHO, 2012. http:/ www.who.int/goe/publications/goe telemedicine 2010.pdf (accessed 10 August 2016).

43. Jarvis-Selinger S, Chan E, Payne R, Plohman K, Ho K. Telemed J E Health 2008:14(7):720-725, https://doi. org/10.1089/tmj.2007.0108

44. Wade VA, Eliott KA, Hiller JE. Clinician acceptance is the key factor for sustainable telehealth services. Qual Health Res 2014;24(5):682-694. https://doi.org/10.1177/1049732314528809

45. McLaren Z, Ardington C, Leibbrandt M. Distance as a barrier to healthcare access in South Africa. A South African labour and development research unit (SALDRU) working paper (97). Cape Town: SALDRU, University of Cape Town, 2013.

46. Hebert MA, Korabek B, Scott R. Moving research into practice: A decision framework for integrating home telehealt into chronic illness care. Int J Med Inform 2006;75(12):786-794. https://doi.org/10.1016/j.ijmedinf.2006.05.041

7. Karr S. Getting to know telepractice. The ASHA leader. 2012;17(30):30-30. https://doi.org/10.1044/leader SCM. 17122012.30

48. Jobson M. Structure of the health system in South Africa. Khulumani Support Group, 2015. https://webcache. googleusercontent.com/search?q=cache:OVSLA4BECq0):https://www.khulumani.net/active (accessed 2 Augus 2016)

Accepted 15 August 2017 